\title{
Controlled Synthesis of Uniform Silver Nanospheres ${ }^{\dagger}$
}

\author{
Hongyan Liang, ${ }^{\ddagger}$ Wenzhong Wang, ${ }^{\ddagger}, \S$ Yingzhou Huang, ${ }^{\ddagger}$ Shunping Zhang, ${ }^{\ddagger}$ Hong Wei, ${ }^{\ddagger}$ and \\ Hongxing $\mathrm{Xu}^{*, \sharp, \|}$ \\ Institute of Physics, Chinese Academy of Sciences, Beijing 100190, P. R China, College of Science, Minzu \\ University of China, Beijing 100081, P. R. China, and Division of Solid State Physics, Lund University, \\ Lund 22100, Sweden
}

Received: November 5, 2009; Revised Manuscript Received: January 11, 2010

\begin{abstract}
We describe a new route, in which the polyethylene glycol (PEG) is used both as a solvent and reducing reagent and the polymer polyvinglpyrrolidone (PVP) is used as capping agent, for the synthesis of monodisperse silver nanoparticles. Uniform nanospheres with an average diameter of $54 \mathrm{~nm}$ can be routinely synthesized in high yield through this approach by using a PVP/AgNO 3 molar ratio of 8 at $260{ }^{\circ} \mathrm{C}$. Both the reaction temperature and the $\mathrm{PVP} / \mathrm{AgNO}_{3}$ molar ratio are crucial for the diameters and size distributions of the nanospheres.
\end{abstract}

\section{Introduction}

Metal nanostructures with functional properties have been extensively studied recently, since they play important roles in many diverse applications. Among the various metal nanostructures, noble metal nanoparticles have attracted considerable attention, due to their unusual optical and chemical properties. Nowadays, a lot of researches have been focused on silver nanoparticles because of their important applications especially in surface-enhanced Raman scattering (SERS). ${ }^{1-8}$ Such properties and applications strongly depend on the morphologies and dimensions of the silver nanostructures. The synthesis of silver nanoparticles with well-controlled morphology and a narrow size distribution is important for uncovering their properties and for achieving their practical applications. During the last two decades, many synthesis methods for the preparation of silver nanoparticles with tailored sizes and shapes have been reported. ${ }^{9-11}$ Using these methods, shape-controlled Ag nanocystals, such as wires ${ }^{12-15}$ rods,${ }^{16}$ plates,${ }^{17}$ cube, ${ }^{18}$ rice,${ }^{19}$ and core-shell bimetallic $\mathrm{Ag} / \mathrm{Au}$ nanoparticles ${ }^{20,21}$ have been routinely synthesized in high yield. However, the fabrication of silver nanospheres with well-controlled sizes in high yield, by a simple and low-cost route, remains a big challenge.

In this paper, we report high-yield synthesis of silver nanospheres by the so-called polyol method, which is convenient, versatile and low cost. To the best of our knowledge, there is still no report so far on the mass synthesis of silver nanospheres based on our present technique. Herein, we find that $\mathrm{Ag}$ nanospheres of dozens of nanometers in size can be easily synthesized by using the polyethylene glycol 600 (PEG $600)$ as solvent and reducing agent, in the presence of polyvinylpyrrolidone (PVP) which acts as capping agent. By controlling and optimizing the synthesis conditions, silver nanospheres with uniform sizes can be obtained.

\footnotetext{
"Part of the "Martin Moskovits Festschrift".

* To whom correspondence should be addressed.

* Institute of Physics.

${ }^{\S}$ Minzu University of China.

"Lund University.
}

\section{Experimental Section}

Materials. PVP with an average molecular weight of 40000 (PVP K30), $\mathrm{AgNO}_{3}$ (purity >99.8 wt \%) and PEG 600 were purchased from Beijing Chemical Reagents Company and used as received. All of them were analytical reagent (AR) grade. Deionized water with resistance of $18 \mathrm{M} \Omega . \mathrm{cm}$ was used in all the cases.

Synthesis of Silver Nanospheres. The silver nanospheres were synthesized by the polyol method. Typically, $444 \mathrm{mg}$ of PVP was added to $40 \mathrm{~mL}$ of PEG with stirring at $80{ }^{\circ} \mathrm{C}$. The function of PVP is protecting the synthesized silver nanospheres from agglomeration. When the solution was transparent, $1 \mathrm{~mL}$ of $0.5 \mathrm{M} \mathrm{AgNO}_{3}$ aqueous solution used as a precursor of $\mathrm{Ag}$ was rapidly injected. During the magnetic stirring and heating process, the color of the solution changed from colorless to light yellow. Then, the reaction solution was transferred to a $80 \mathrm{~mL}$ Teflon-sealed autoclave and heated at $260{ }^{\circ} \mathrm{C}$ for $24 \mathrm{~h}$. After the reaction was completed, the solution was cooled in air to room temperature.

The purification of the silver nanospheres was conducted by centrifugation. They are separated from the surfactants and PEG by centrifugation at $16000 \mathrm{rpm}$ for $20 \mathrm{~min}$. The obtained precipitates were then dissolved in deionized water or ethanol and centrifuged several times again to remove the PEG and PVP. Finally, the sample was dispersed in ethanol for further characterization.

Instrumentation. Scanning electron microscopy (SEM) images were obtained using a Hitach1 S-4800 scanning electron microscope. Analysis was performed on samples deposited onto silicon supports. Transmission electron microscopy (TEM) and selected area electron diffraction (SAED) studies were performed with a Philips CM-12 microscope operated at an accelerating voltage of $100 \mathrm{kV}$. Samples were prepared by dropping the particles in ethanol on copper grids supported carbon film. UV-vis spectroscopy was carried out on U-3010 Spectrophotometer with a spectral range from 900 to $250 \mathrm{~nm}$. Powder X-ray diffraction (XRD) measurements were performed on a Bruker D8 Discover with GADDS and $\mathrm{Cu}$ KR radiation at $\lambda=0.15418 \mathrm{~nm}$. 


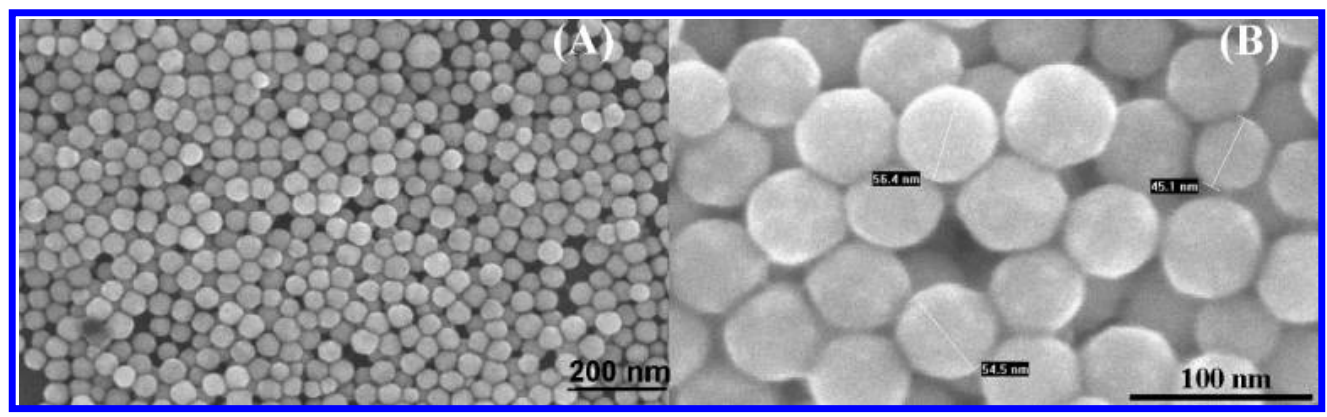

Figure 1. (A) Low- and (B) high-magnification SEM images of the silver nanospheres prepared at $260{ }^{\circ} \mathrm{C}$ for $24 \mathrm{~h}$ with molar ratio of PVP to $\mathrm{AgNO}_{3}$ of 8 .

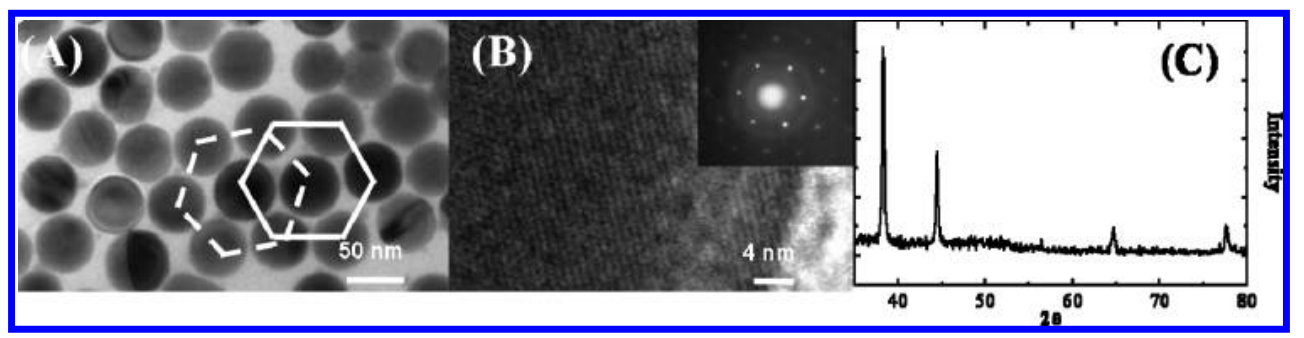

Figure 2. (A) TEM and (B) HRTEM images of the silver nanospheres prepared at $260{ }^{\circ} \mathrm{C}$ for $24 \mathrm{~h}$ with molar ratio of $\mathrm{PVP}$ to $\mathrm{AgNO} \mathrm{B}_{3}$ of 8 , and the SAED pattern (inset) of an individual silver nanosphere with a diameter of about $50 \mathrm{~nm}$. (C) Powder XRD pattern taken from the same batch of sample.

\section{Results and Discussion}

Synthesis of Silver Nanospheres. SEM images show that the as-prepared products $\left(260{ }^{\circ} \mathrm{C}, 24 \mathrm{~h}\right)$ are regular spherical particles with uniform size without sharp or truncated corners as illustrated in Figure 1A. The high-magnification image (Figure 1B) demonstrates that these nanospheres have smooth surfaces and a mean diameter of $54 \mathrm{~nm}$, with a standard deviation of $10 \mathrm{~nm}$.

TEM and high-resolution TEM (HRTEM) images of the same sample are shown in Figure 2. Figure 2A shows that all of the silver nanospheres are circular. Interestingly, some of them selfassembled into ordered two-dimensional (2D) hexagonal patterns on the carbon film when the TEM sample was prepared, as marked with white hexagons in Figure 2A. HRTEM images have given us further insight into the microstructure and crystallinity of the as-prepared silver nanospheres (Figure 2B). The clear lattice fringes confirm that the sphere is highly crystallized. The lattice spacing of $0.232 \mathrm{~nm}$ corresponds to the $\langle 111\rangle$ planes of Ag. The results show that the dominant faces of the silver spheres are $\langle 111\rangle$. The SAED pattern was obtained by directing the electron beam perpendicular to one of the spheres. The hexagonal symmetry diffraction spots pattern shown in the inset of Figure 2B confirmed that the sphere is well crystalline, and its face is indexed to $\langle 111\rangle$ planes. Both HRTEM images and SAED pattern can confirm that the silver sphere is single-crystal. In fact, more than $60 \%$ of spheres synthesized in this condition are single-crystal. XRD pattern was shown in Figure 2C, four peaks can be observed, corresponding to diffractions from the (111), (200), (220), and (311) planes of face-centered-cubic (fcc) phase which indicates that the Ag particles are well crystallized.

The sizes and size distributions of silver nanospheres have a strong dependence on the reaction conditions, such as reaction temperature and molar ratio of the repeating unit of PVP to $\mathrm{AgNO}_{3}$.

Temperature Effect. By simply changing the reaction temperature, the size and shape can be significantly tuned. For example, the products were dominated by silver nanospheres by applying high temperature from 200 to $260^{\circ} \mathrm{C}$. In contrast, lower temperatures were not beneficial to the formation of silver nanospheres. Figure 3A shows SEM images of the silver nanocrystals prepared at $260^{\circ} \mathrm{C}$, indicating that the as-prepared nanocrystals have spherical shape with uniform size distribution (an average diameter of $54 \mathrm{~nm}$ with a size distribution ranging from 39 to $64 \mathrm{~nm}$ ) as shown in Figure 3B. Compared to 260 ${ }^{\circ} \mathrm{C}$, the $\mathrm{Ag}$ nanocrystals only have very few irregular shapes when the reaction temperature was set to $220{ }^{\circ} \mathrm{C}$, and have a wide size distribution in the range of $24-49 \mathrm{~nm}$ with an average diameter of $39 \mathrm{~nm}$ as shown in Figure 3, panels C and D. At $200{ }^{\circ} \mathrm{C}$, the as-prepared nanoparticles were mainly composed of nanospheres, but a few irregular particles with sharp corners were presented which was shown in Figure 3E. The size distribution of the $\mathrm{Ag}$ nanocrystals is in the range of 27 to 73 $\mathrm{nm}$ with the average diameter of $49 \mathrm{~nm}$ (Figure 3F). When the reaction temperature was further decreased to $180{ }^{\circ} \mathrm{C}$, the products were mainly composed of quasi-spherical (including some sharp or truncated corners) Ag nanoparticles (Figure 3G). These nanocrystals have an average diameter of $70 \mathrm{~nm}$ with wider size distribution from 25 to $125 \mathrm{~nm}$ as illustrated in Figure $3 \mathrm{H}$. Below $180^{\circ} \mathrm{C}$, however, the products were dominated by irregular silver particles, and nearly without regular Ag nanospheres (data not shown). It is worth pointing out that nearly all of the irregular silver particles obtained at temperature lower than $260{ }^{\circ} \mathrm{C}$ are twinned structure.

The sizes and size distributions of $\mathrm{Ag}$ nanocrystals which were synthesized at different temperatures were further confirmed by the UV/vis spectra. As shown in Figure 3I, the Ag nanospheres prepared at $260^{\circ} \mathrm{C}$ exhibits an extinction peak at $413 \mathrm{~nm}$ with a narrow full width at half-maximum (cyan line in Figure 3I) which is consistent with the SEM result (Figure 3 , panels $\mathrm{A}$ and $\mathrm{B}$ ). The extinction peak of $\mathrm{Ag}$ nanocrystals prepared at $220{ }^{\circ} \mathrm{C}$ is around $400 \mathrm{~nm}$ (red line in Figure 3I) which has a $13 \mathrm{~nm}$ blue-shift compared to the $260^{\circ} \mathrm{C}$ samples. The smaller size of Ag nanocrystals, the average diameter decreased from 54 to $39 \mathrm{~nm}$, contributes to the blue shift of 13 $\mathrm{nm}$. When the temperature was decreased to $200{ }^{\circ} \mathrm{C}$, the 


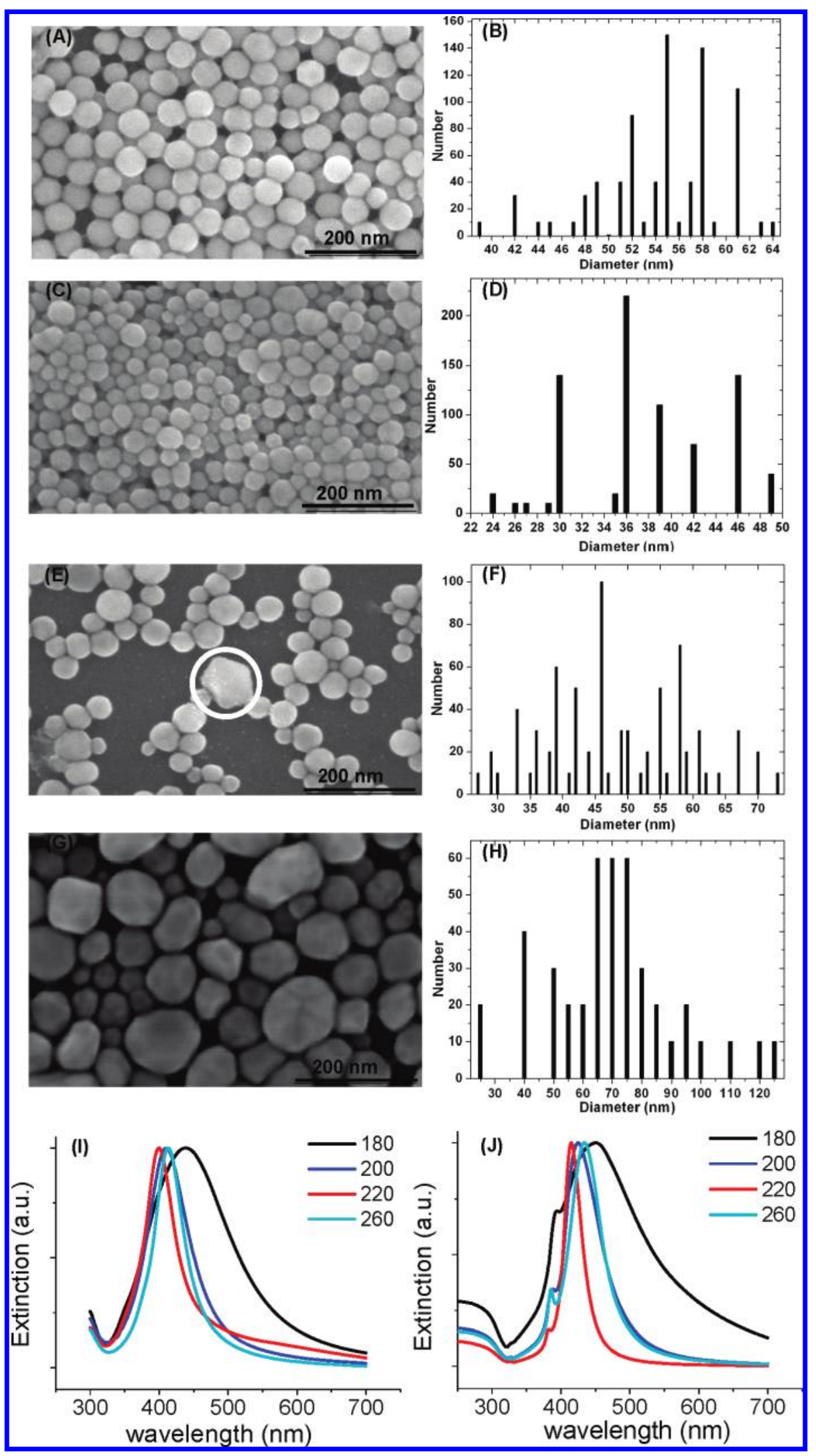

Figure 3. $(\mathrm{A}-\mathrm{H})$ The SEM images and the corresponding histograms for the size distribution of the silver nanocrystals obtained at different temperatures. (A and B) $260{ }^{\circ} \mathrm{C},\left(\mathrm{C}\right.$ and D) $220^{\circ} \mathrm{C}$, (E and F) $200^{\circ} \mathrm{C}$, (G and H) $180{ }^{\circ} \mathrm{C}$. (I and J) Experimentally measured and theoretically calculated UV/vis extinction spectra of the silver nanocrystals prepared at different temperatures: cyan line $\left(260{ }^{\circ} \mathrm{C}\right)$, red line $\left(220{ }^{\circ} \mathrm{C}\right)$, violet line $\left(200{ }^{\circ} \mathrm{C}\right)$, and black line $\left(180^{\circ} \mathrm{C}\right)$.

extinction peak is slightly bule-shifted and a little bit wider (violet line in Figure 3I) in comparison with the $260{ }^{\circ} \mathrm{C}$ samples. The wider peak width is due to the larger size distribution as can be seen in Figure 3, panles E and F. The extinction peak of the Ag nanocrystals prepared at $180{ }^{\circ} \mathrm{C}$ red-shifts to $440 \mathrm{~nm}$ (black line in Figure 3I) and the extinction band is much broader 


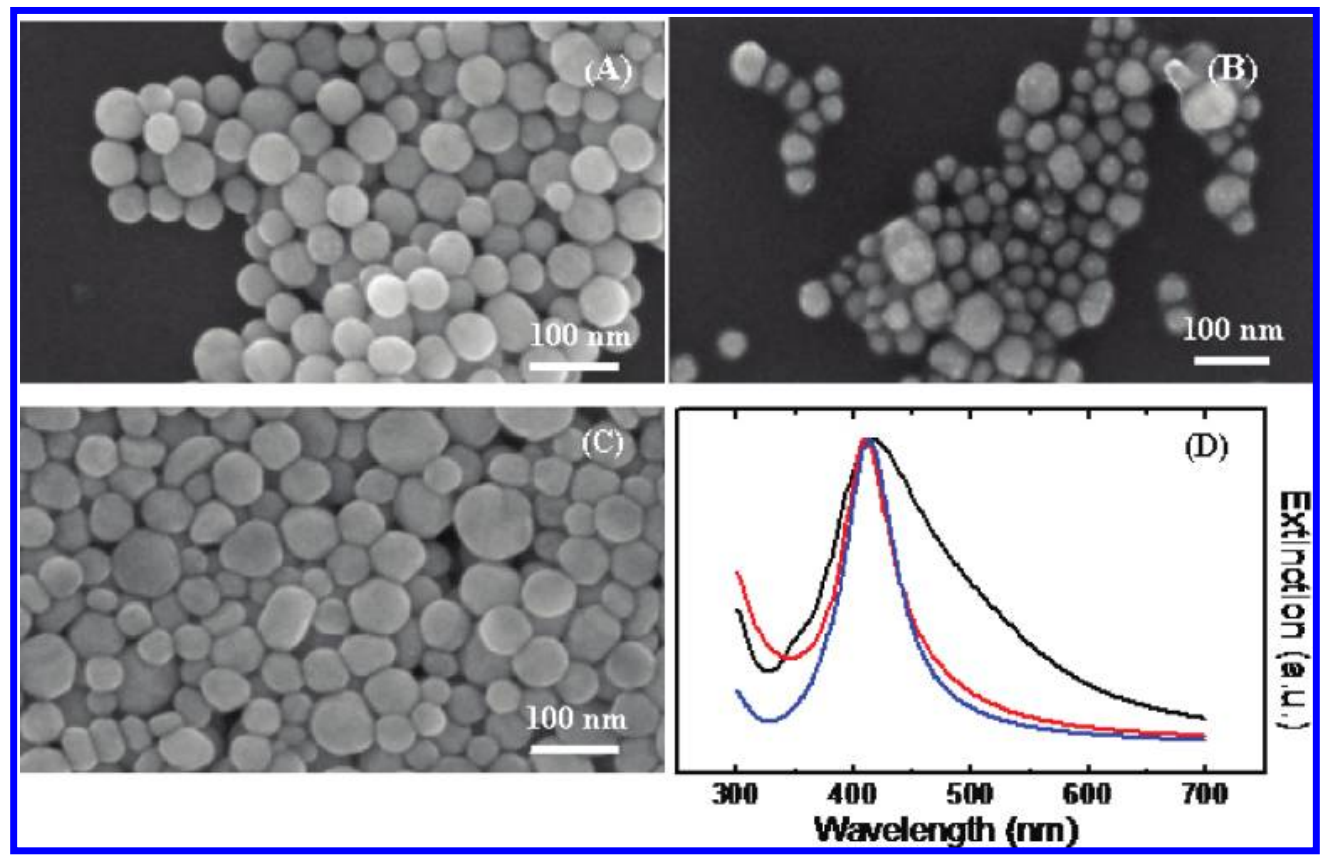

Figure 4. The SEM images of the silver nanocrystals synthesized with different molar ratios of PVP to $\mathrm{AgNO}_{3}$. (A) 8, (B) 16, and (C) 4. (D) $\mathrm{UV} /$ vis extinction spectra of the silver nanocrystals prepared with different molar ratios of PVP to $\mathrm{AgNO}_{3}$ : cyan line (8), red line (16), and black line (4).

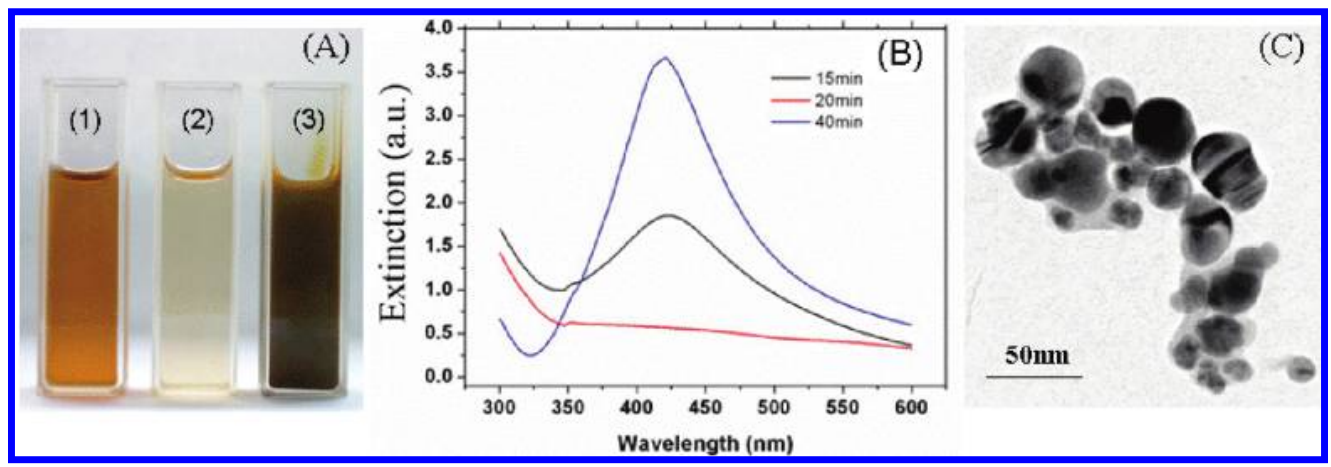

Figure 5. (A) Photos of solution prepared with different time at $260{ }^{\circ} \mathrm{C}$. (1) 15 , (2) 20 , and (3) 40 min. (B) Corresponding UV/vis spectra of the as-prepared solution. Black line $(15 \mathrm{~min})$, red line $(20 \mathrm{~min})$, and blue line $(40 \mathrm{~min})$. (C) TEM images of silver particles prepared at $260{ }^{\circ} \mathrm{C}$ for 15 $\min$.

due to the big size and wide size distribution of the nanoparticles, which is in accordance with the SEM image (Figure 3G).

To theoretically confirm the experimental results, the extinction cross section is calculated through Mie theory, which is proved to predict the SPR of spherical NPs. ${ }^{22}$ Inhomogeneous broadening from size distribution of each sample is mimicked by a weighting factor determined directly from the statistics histogram. Dielectric constants of Ag are taken from ref. ${ }^{23}$ Well agreements between the calculated and measured results are achieved, as can be seen in Figure 3J. A small shoulder, attributed to quadrupole plasmon resonance, appears in each calculated spectrum but is absent in the experimental results. This is probably due to a nonspherical morphology distribution in the experiment which is not considered in the Mie calculations.

Molar Ratio Effect. Although PVP have been used as reducing agents in aqueous solution, ${ }^{24,25}$ in this polyol method, PVP works as capping agents in PEG solution. In absence of the PVP, we also could obtain silver nanoparticles, however, without the capping agent, the size and shape will be not uniform. This also confirms that the PEG played roles as reducing reagent. By adjusting the molar ratio between the repeating unit of PVP and $\mathrm{AgNO}_{3}$, the thickness of PVP coating and the location of PVP chains on the surface of a seed could both be modified, which in turn, altered the growth of silver nanostructures. ${ }^{26}$ So, the molar ratio between the repeating unit of PVP and $\mathrm{AgNO}_{3}$ was also crucial. When the molar ratio was kept at 8, the large quantity and good uniformity of silver nanospheres were achieved as shown in Figure 4A. However, if the molar ratio was increased from 8 to 16 , smaller spheres with an average diameter of $38 \mathrm{~nm}$ and a wide size distribution of 21 to $74 \mathrm{~nm}$ were obtained (Figure 4B). When the molar ratio was decreased to 4 , the as-prepared products were composed of nanospheres and some irregular nanoparticles as shown in Figure 4C. The nanocrystals have an average diameter of about $61 \mathrm{~nm}$ with a wider diameter distribution ranging from 30 to $95 \mathrm{~nm}$. The sizes and size distributions also further confirmed by the UV/vis extinction spectra (Figure 4D). There is no significant difference between the spectra for the samples obtained at the molar ratio of 8 and 16. However, when the molar ratio was decreased to 4 , wider extinction was shown which is due to the wider size distribution. By comparing the products shown in Figure 4, it can be concluded that neither increase nor decease of the molar ratio of PVP to $\mathrm{AgNO}_{3}$ is favorable for the formation of uniform silver nanospheres.

Growth Process. To further understand the growth mechanism of the silver nanospheres, the growth process was studied 
by time-dependent investigations of color changes and the corresponding UV/vis extinction spectra for the reaction solution. In Figure 5, the color changes and the corresponding UV/ vis spectra for the reaction solution at three different reaction time from a reaction system that was kept at $260{ }^{\circ} \mathrm{C}$ are shown. The solution was directly used for the UV/vis measurement without any further purification or dilution. When the solution was heated at $260{ }^{\circ} \mathrm{C}$ for $15 \mathrm{~min}$, it appears yellow which is further changed almost colorless at $20 \mathrm{~min}$ and changed to silver gray after $40 \mathrm{~min}$, as is shown in Figure 5A. For these three solution samples, UV/vis spectral measurements were performed. The yellow solution (15 $\mathrm{min}$ ) exhibits a characteristic peak at about $410 \mathrm{~nm}$ as shown in Figure 5B (black line). One can find that the peak is very broad, which is caused by the large size distribution of Ag nanoparticles as shown in TEM image in Figure 5C. The peak is also very weak, indicating that the concentration of the silver nanoparticles is low. The UV/vis spectrum taken from the colorless solution does not exhibit any peak of the silver nanoparticles as shown in Figure 5B (red line), which indicates that those initially formed silver nanoparticles are dissolved into the PEG solution. When the solution was kept at $260{ }^{\circ} \mathrm{C}$ for $40 \mathrm{~min}$, the color of the solution is silver gray, indicating that a large amount of silver nanoparticles are formed, which is confirmed by corresponding UV/vis spectrum that exhibits a much stronger characteristic peak at about $410 \mathrm{~nm}$ (blue line in Figure 5B) than the first synthesis stage at $15 \mathrm{~min}$. Comparing the sharp peak in Figure 3I after much longer time reaction at the same temperature, the extinction peak at $40 \mathrm{~min}$ is broader. It is reasonable that the small particles formed at the initial stages can still grow bigger, while the larger particles could be partly dissolved to become smaller. Finally, all the particles become nearly uniform in size, because there could exist a lowest surface energy for nanoparticles with a certain size. In this particular case at the reaction temperature $260{ }^{\circ} \mathrm{C}$, the size is $54 \mathrm{~nm}$ in the diameter.

On the basis of the above experimental results and analysis, we propose a possible mechanism for the formation of silver nanospheres. Probably, the growth of sliver nanosphere involves a multistep process: at the initial stage, single-crystal seeds and twinned seeds can be formed through homogeneous nucleation which can be confirmed by Figure 5C. Then these initially formed twinned nanoparticles, which are more reactive and unstable, ${ }^{27}$ are dissolved into solution. So the color of the solution changed from yellow to colorless. With time going on, the silver ions in the colorless solution reached the supersaturation state, and they start to nucleate and grow quickly again.

The growth processes at different temperature are similar. However, the reducing power of PEG has a strong dependence of reaction temperature. At lower temperature, it is possible that the formation of twinned seeds is more preferential and the dissolution of twinned particles is not as efficient as at $260{ }^{\circ} \mathrm{C}$. So the products at low temperature are dominated by irregular twinned particles. But at $260^{\circ} \mathrm{C}$, most nanoparticles grow from single-crystal seeds, and finally become uniform in size (54 nm) probably because of the lowest surface energy at that size.

\section{Conclusions}

In summary, a new way to synthesize silver nanospheres with uniform diameters in high yields is described, which is based on the use of PEG which acts as both a solvent and a reducing reagent and PVP as a capping agent. Uniform nanospheres with an average diameter of $54 \mathrm{~nm}$ could be routinely synthesized through this approach by using a molar ratio of 8 between the repeating unit of PVP and $\mathrm{AgNO}_{3}$ at $260{ }^{\circ} \mathrm{C}$. The morphology and dimensions of the silver nanoparticles can be easily modulated by controlling the synthesis temperature and the molar ratio of PVP to $\mathrm{AgNO}_{3}$. These silver nanoparticles should find potential applications in a variety of areas.

Acknowledgment. This work was supported by NSFC Grant No. 10625418, 10874233, MOST Grant No. 2006DFB02020, 2007CB936800, 2009CB930700, "Bairen Project" of CAS, and Research Foundation of The State Ethnic Affairs Commission of PRC (No. 07ZY02).

\section{References and Notes}

(1) Moskovits, M. Rev. Mod. Phvs. 1985, 57, 783.

(2) Moskovits, M.; Tay, L. L.; Yang, J.; Haslett, T. Top. App. Phvs. 2002, 82, 215.

(3) Moskovits, M. J. Raman Spectrosc. 2005, 36, 485-496.

(4) Xu, H. X.; Bjerneld, E. J.; Kall, M.; Borjesson, L. Phvs. Rev. Lett. 1999, $83,4357$.

(5) Xu, H. X.; Aizpurua, J.; Kall, M.; Apell, P. Phvs. Rev. E 2000, 62, $4318-4324$.

(6) Talley, C. E.; Jackson, J. B.; Oubre, C.; Grady, N. K.; Hollars, C.; Lane, S. M.; Huser, T. R.; Nordlander, P.; Halas, N. J. Nano Lett. 2005, 5, 1569.

(7) Braun, G.; Pavel, I.; Morrill, A. R.; Seferos, D. S.; Bazan, G. C.; Reich, N. O.; Moskovits, M. J. Am. Chem. Soc. 2007, 129, 7760.

(8) Shegai, T.; Li, Z.; Dadosh, T.; Zhang, Z.; Xu, H. X.; Haran, G. Proc. Natl. Acad. Sci. U.S.A. 2008, 105, 16448.

(9) Chen, S.; Carroll, D. L. Nano Lett. 2002, 2, 1003.

(10) Maillard, M.; Giorgio, S.; Pileni, M.-P. Adv. Mater. 2002, 14, 1084.

(11) Pastoriza-Santos, I.; Liz-Marzán, L. M. Nano Lett. 2002, $2,903$.

(12) Sun, Y. G.; Xia, Y. N. Adv. Mater. 2002, 14, 833.

(13) Sun, Y. G.; Xia, Y. N. J. Am. Chem. Soc. 2004, 126, 3892.

(14) Sun, Y. G.; Gates, B.; Mayers, B.; Xia, Y. N. Nano Lett. 2002, 2 , 165 .

(15) Sun, Y. G.; Yin, Y. D.; Mayers, B. T.; Herricks, T.; Xia, Y. N. Chem. Mater. 2002, 14, 4736.

(16) Jana, N.; Gearheart, L. Murphy. J. Chem. Commun. 2001, 617.

(17) Sun, Y. G.; Xia, Y. N. Adv. Mater. 2003, 15, 695.

(18) Sun, Y. G.; Xia, Y. N. Science. 2002, 298, 2176.

(19) Liang, H. Y.; Yang, H. X.; Wang, W. Z.; Li, J. Q.; Xu, H. X. M. J. Am. Chem. Soc. 2009, 131, 6089.

(20) Moskovits, M.; Srnova-Sloufova, I.; Vlckova, B. J. Chem. Phvs. 2002, 116, 10435.

(21) Srnova-Sloufova, I.; Lednicky, F.; Gemperle, A.; Gemperlova, J. Langmuir 2000, 16, 9928.

(22) Kreibig, U.; Vollmer, M. Optical Properties of Metal Clusters; Springer-Verlag: Berlin, 1995.

(23) Johnson, P. B.; Christy, R. W. Phvs. Rev. B 1972, 6 (12):), 43704379

(24) Xiong, Y. J.; Washio, I.; Chen, J. Y.; Cai, H. G.; Li, Z. Y.; Xia, Y. N. Langmuir 2006, 22, 8563.

(25) Washio, I.; Xiong, Y. J.; Yin, Y. D.; Xia, Y. N. Adv. Mater. 2006, 18,1745 .

(26) Wiley, B.; Sun, Y. G.; Mayers, B.; Xia, Y. N. Chem.-Eur. J. 2005, 11,454 .

(27) Lu, X. M.; Rycenga, M.; Skrabalak, S. E.; Wiley, B.; Xia, Y. N. Annu. Rev. Phvs. Chem. 2009, 60, 167.

JP9105713 